\title{
UV and optical quantitative spectroscopy of early B-type supergiants in M 33
}

\author{
Miguel A. Urbaneja ${ }^{1}$, Artemio Herrero ${ }^{1,2}$, Daniel J. Lennon ${ }^{3}$, F. \\ Najarro $^{4}$, Joachim Puls ${ }^{5}$, Stephen J. Smartt ${ }^{6}$, and Rolf-Peter Kudritzki ${ }^{7}$ \\ ${ }^{1}$ Instituto de Astrofísica de Canarias, \\ C/ Vía Láctea s/n, E-38200 La Laguna, Tenerife, España \\ ${ }^{2}$ Departamento de Astrofísica, Universidad de La Laguna, \\ Avda. Astrofísico Francisco Sánchez, s/n, E-38071 La Laguna, España \\ ${ }^{3}$ The Isaac Newton Group of Telescopes, Apartado de Correos 321, \\ E-38700, Santa Cruz de La Palma, Canary Islands, España \\ ${ }^{4}$ Instituto de Estrucutra de la Materia, CSIC, \\ Serrano 121, E-28006, Madrid, España \\ ${ }^{5}$ Universitätssternwarte, Scheinerstraße 1, D-81679 München, BRD \\ ${ }^{6}$ Institute of Astronomy, University of Cambridge, \\ Madingley Road, Cambridge CB3 OHA, UK \\ ${ }^{7}$ Institute for Astronomy, University of Hawaii at Manoa, \\ 2680 Woodlawn Drive, Honolulu, HI 96822, USA
}

\begin{abstract}
We present the analysis of UV and optical spectra of M33 early Btype supergiants. Stellar parameters, metal abundances ( $\mathrm{Si}, \mathrm{Mg}, \mathrm{O}$ and $\mathrm{N}$ ) and mass loss rates are derived from anlyses of optical spectra by means of non-LTE unblanketed unified model atmospheres (FASTWIND code, Santolaya-Rey, Puls $\&$ Herrero 1997). These analyses use wind terminal velocities previously derived from the analysis of UV P-Cygni profiles. Stellar radial gradients of Si, O and $\mathrm{Mg}$ are derived for the M33 disk. Differential abundances of those elements with respect to Galactic counterparts are also presented, along with nitrogen. Finally, the wind momenta of M 33 stars is derived and compared with the one found for Galactic B-type supergiants.
\end{abstract}

\section{Discussion}

Quantitative spectroscopy of stars has become a powerful tool in the study of chemical composition of external galaxies. Among the normal population of a galaxy, B-type supergiants are suitable objects for chemical abundance analyses, because their blue spectra are rich in metal absorption lines, which together with their intrinsic high luminosities allow the study of such stars at large distances. In addition, these objects provide a new tool for the determination of extragalactic distances by means of the so-called Wind momentum - Luminosity Relationship (WLR). Before applying a WLR, it must be calibrated for the effects of metallicity and for the different spectral types (Kudritzki et al. 1999).

This contribution presents our results on the metal content of several early B-type supergiants in M 33, as well as the comparison of the wind of these stars with their Galactic counterparts. This work is aimed at tracing the distribution 
of several elements across the disk of M 33 and to determine a consistent distance to that galaxy, using stars of different metallicities, that constitutes a strong test of the WLR method. Compared to their Galactic counterparts, M 33 early Btype supergiants show a 'CNO Galactic abundance pattern', meaning that the degree of chemical evolution is very similar, within the uncertainties. The differential stellar abundances of the $\alpha$-elements are well correlated; within the uncertainties, the analyzed M 33 B-type supergiants seem not to show contamination in their atmospheres by ON-cycle processed material. Derived stellar absolute $\mathrm{O}$ abundances are also in agreement with the nebular results from Vilchez et al. (1988), while the derived $\mathrm{N}$ abundances are larger, being the difference larger for lower metallicities; there are several ways to product such effect (depletion onto dust grains, CN-cycle processed material). Given our present uncertainties, we can rule out none of both processes. The metallicity effects can be observed by comparing their modified wind momenta with those expected from the work by Kudritzki et al. (1999). For a complete discussion of the analysis and results see Urbaneja et al. (2002; in preparation).

\section{References}

Kudritzki, R.-P., Puls, J., Lennon D.J., et al. 1999, A\&A 350, 970

Santolaya-Rey, A.E., Puls, J., Herrero, A. 1997, A\&A 323, 488

Urbaneja, M.A., Herrero, A., Kudritzki, R.-P., et al. 2002, A\&A 386, 1019

Vílchez, J.M., Pagel, B.E.J., Díaz, A.I., et al. 1998, MNRAS , 135, 633

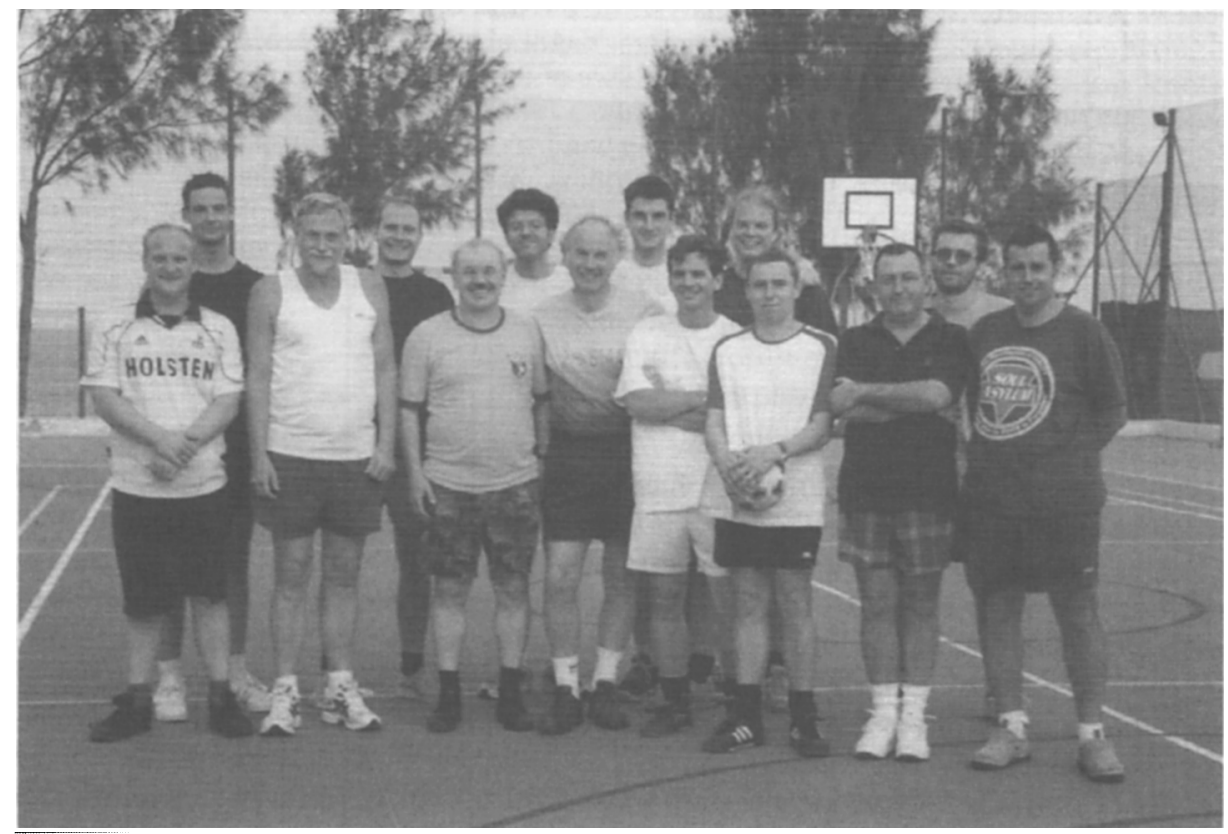

The winning team: Norris, Abbott, Kudritzki, Vink, Zinnecker, Dessart, Gieren, Evans, Lennon, Henley, Smartt, Herrero, Girardi, and Najarro 